\title{
PRODUÇÃO DO CONHECIMENTO EM GINÁSTICA: UMA ANÁLISE A PARTIR DOS PERIÓDICOS BRASILEIROS
}

\author{
LA PRODUCCIÓN DEL CONOCIMIENTO EN GIMNASIA: UN ANÁLISIS DE LOS \\ PERIÓDICOS BRASILEÑOS
}

KNOWLEDGE PRODUCTION IN GYMNASTICS: AN ANALYSIS BASED ON

BRAZILIAN JOURNALS

Michele Viviene Carbinatto*, Aline Dessupoio Chaves ${ }^{\star *}$, Wagner Wey Moreira**, Ana Laura Souza de Castro Coelho**, Regina Maria Rovigati Simões ${ }^{* \star}$

\begin{abstract}
Palavras-chave Bibliometria.

Pesquisa qualitativa. Indicadores de ciência, tecnologia e inovação.

Ginástica.

Resumo: Este artigo, de caráter descritivo do tipo bibliográfico, analisou o cenário (estado da arte) da ginástica nas pesquisas acadêmico-científicas publicadas em periódicos nacionais indexados na área da Educação Física pela Capes entre os anos de 2000 e 2015. Detectaram-se 340 trabalhos distribuídos em 61 revistas, com prevalência na Região Sudeste e destaque de publicação sobre o tema no ano de 2010. Ademais, observou-se que a produção nacional é socializada, sobretudo em revistas de estratos B4, B1 e B2, e que nenhuma das revistas divulgou continuamente estudos sobre ginástica no período analisado. É possível confirmar que a elevação de publicações sobre ginástica coincidiu com a organização e consolidação de eventos na área e que a redução de produções após o ano 2010 pode ser explicada pela busca da internacionalização das pesquisas e interesse de publicação em revistas de maior fator de impacto.
\end{abstract}

Palabras clave

Bibliometría. Investigación

cualitativa.

Indicadores de

ciencia, tecnología

e innovación.

Gimnasia.

Resumen: Este artículo, de carácter descriptivo bibliográfico, analizó el escenario (estado del arte) de la gimnasia en las investigaciones académico-científicas publicadas en revistas brasileñas indexadas en el área de Educación Física por la Capes entre 2000 y 2015. Se detectaron 340 trabajos distribuidos en 61 revistas, con predominancia de la región sudeste y destaque de publicación sobre el tema en el año 2010. Además, se observó que la producción nacional es socializada, sobre todo en revistas de los estratos B4, B1 y B2, y que ninguna de las revistas divulgó continuamente estudios sobre gimnasia en el período analizado. Es posible afirmar que el incremento de publicaciones sobre gimnasia coincidió con la organización y consolidación de eventos en el área y que la reducción de la producción a partir del año 2010 puede explicarse por la búsqueda de la internacionalización de la investigación y el interés por publicar en revistas de mayor factor de impacto.

Abstract: Based on bibliographic parameters, this article analyzed the current scenario of "gymnastics" in academic-scientific research published in Brazilian journals indexed in the area of Physical Education by Capes in 2000-2015. We found 340 articles on 61 journals, mainly from Southeastern Brazil, with a peak in publication on the subject in 2010. Furthermore, Brazilian production is conveyed in journals from strata B4, B1 and B2, and none of them continuously published studies about gymnastics during the period. It is possible to confirm that the increase in publications coincided with the organization and consolidation of events in the area. A decrease in production after $2010 \mathrm{can}$ be explained by the attempt to internationalize research and high interest in publishing in journals of higher impact factor.
* Universidade de São Paulo. São Paulo, SP, Brasil.

E-mail: mcarbinatto@usp.br

** Universidade Federal Do Triângulo Mineiro. Uberaba, MG, Brasil. E-mail: alinedessupoio@uftm.edu.br

Recebido em: 29-12-2015 Aprovado em: 20-09-2016 (c) (i) (8) Licence 


\section{INTRODUÇÃO}

O conhecimento científico se caracteriza por envolver uma atitude investigativa que busca revelar e compreender fatos que ainda não são vistos claramente, e as produções acadêmicas são ferramentas utilizadas na produção e disseminação daquele. Por sua vez, 0 desenvolvimento da ciência e das modernas tecnologias de informação nas últimas décadas resultou na criação de um conjunto de periódicos científicos com o objetivo de expandir e disseminar o conhecimento de qualidade produzido pelos pesquisadores (TANI, 2014; ROSA; LETA, 2010; BETTI et al., 2004).

Atualmente, os periódicos podem voltar-se às publicações em uma determinada temática (ex.: lazer, terceira idade) ou a uma área de conhecimento específica (ex.: Psicologia, Educação Física). Independentemente do desequilíbrio numérico, é fato que encontramos periódicos para todas as possibilidades de pesquisa, com política editorial, periodicidade na publicação e estruturas de distribuição e comercialização do seu produto (TANI, 2014).

Como consequência, as profissões academicamente orientadas, como 0 caso dos cursos de graduação em Educação Física e/ou Esporte, visam organizar, sistematizar e propor um corpo de conhecimentos acadêmico-científicos que auxilie na intervenção profissional (TANI, 2011) e que será publicado em formato de livros, capítulos de livros, artigos, palestras, vídeos expositivos, dentre outros.

Essa diversidade de divulgação do conhecimento é desafiada pela dificuldade em identificar as especificidades e tempo disponível para estudo (READE; RODGES; HALL, 2008) e, por este motivo, trazer à tona onde se encontram a maior parte dos artigos referentes a uma temática específica pode sanar, ao menos num primeiro momento, aquelas barreiras.

Apesar de a análise bibliométrica ter crescido consideravelmente nos últimos anos, pois permite visualizar tendências e projeções de um determinado assunto (BASKURT, 2011) e refletir mudanças em diversos níveis (GOKCEOGLU; OKAY; SEZER, 2008), esse método ainda é incipiente na Educação Física e Esporte, considerando a abrangência e as possibilidades que a área possui (MATOS et al., 2013; SOUZA; MARCHI JUNIOR, 2011).

Inserida no universo da área em questão, encontra-se a ginástica, que, além de ser considerada um conhecimento clássico (METODOLOGIA..., 1992), tem sua história entrelaçada com a da Educação Física e do Esporte. Ela representa uma parte fundamental da formação e da atuação profissional e provavelmente é o conhecimento que mais recebeu influências dos modismos, ganhando roupagens diferentes de acordo com as épocas, as culturas e os interesses distintos, com respaldo direto na sua forma de investigação e de intervenção (TIBEAU, 1999; RINALDI, 2005).

Além disso, encontramos análises pouco abrangentes sobre a produção acadêmica em relação à ginástica no Brasil (MILANI; SOARES; BORTOLETO, 2015; LIMA et al., 2015; SAMPAIO; KRAESKI; FARIAS, 2015; TANNÚS; SIMÕES, 2015a; 2015b; SILVA et al., 2015; FERREIRA; TEIXEIRA, 2014; MATIOLI; TEIXEIRA; RINALDI, 2014).

Com 0 intuito de viabilizar a comunicação e divulgar os conhecimentos produzidos entre e pelos pares de uma comunidade científica (TANI, 2014), este artigo analisa a produção sobre o tema "ginástica" nos periódicos nacionais entre os anos de 2000 e 2015. 
Neste trabalho focalizamos a análise a partir de algumas problemáticas: característica do periódico em relação ao seu estrato, procedência territorial, quantitativo de artigos publicados por estrato e anuidade.

\section{MÉTODO}

Textos sobre o estado da arte são de caráter bibliográfico e contribuem para a sistematização e avaliação da produção acadêmica em determinada área do conhecimento em período previamente estabelecido e vislumbram responder aspectos e dimensões que devem ser elucidados (FERREIRA NETO, 2002).

Para tanto, utilizamos para análise os indicadores bibliométricos (MUGNANI; CARVALHO; CAMPANATTI-ORTIZ, 2006) de revistas e artigos sobre ginástica publicados em periódicos nacionais nos estratos $\mathrm{A} 1$ a $\mathrm{C}$ cadastrados no sistema Web-Qualis da área 21, do ano 2000 a junho de 2015, em duas etapas: a. Coleta e organização dos dados e b. Categorização e análise quantitativa e qualitativa.

A opção pelos periódicos se deu pelos critérios de qualidade para a divulgação das pesquisas propostas por aqueles que contribuem para a definição e legitimidade na área (TANI, 2014).

Para o desenvolvimento da investigação foi feito um levantamento online de todas as revistas da área de Educação Física classificadas e disponíveis na base de dados do site oficial da Coordenação de Aperfeiçoamento de Pessoal de Nível Superior (Capes), no sistema WebQualis, 2007-2009/20010-2012/2013-atual, o qual estabelece periodicamente a estratificação (COORDENAÇÃO DE APERFEIÇOAMENTO DE PESSOAL DE NÍVEL SUPERIOR, 2012). Os artigos publicados entre 2000 e 2006 não receberam estratificação.

Em todas as buscas foram utilizados os seguintes critérios de inclusão: revistas nacionais com o idioma português, revistas eletrônicas que possuíam ISSN (International Standard Serial Number -Número Internacional Normalizado para Publicações Seriadas) e publicadas entre o ano 2000 e junho de 2015, objetivando analisar o cenário da publicação na área da ginástica no século XXI.

A opção por um banco de dados virtual justifica-se por possibilitar boa visibilidade, pela facilidade de acesso, por garantir maior abrangência das pesquisas mais recentes sobre o tema e por tratar de informações disponíveis a qualquer profissional da área. Além disso, no levantamento de 1970 a 2012 na base de dados Web of Knowledge, a maioria (63\%) das publicações é em periódicos nacionais (ANDRADE et al., 2013), o que confirma que nossa pesquisa pode trazer um importante retrato da realidade sobre a ginástica.

No primeiro levantamento obtivemos um total de 1981 revistas, sendo 518 nacionais na área da Educação Física. Após essa etapa passamos a identificar os artigos presentes nos periódicos e que tivessem no título ou nas palavras-chave os seguintes descritores: ginástica, academia, fitness e ginasta.

Estudos apontam que a utilização das palavras-chave em um artigo potencializa 0 acesso ao seu conteúdo para além da informação refletida no título e resumo e, normalmente, elas se referem ao alcance de um tema e seus conceitos primordiais. Além disso, o uso de palavras-chave é útil na indexação, pesquisa e categorização da investigação (TONELLO; 
LUNARDELLI; ALMEIDA JÚNIOR, 2012; ERCAN; CICEKLI, 2007). A partir do levantamento no banco de dados, 389 revistas não tinham artigos que atendiam aos critérios, 43 não foram encontradas, 14 não permitiam que o site fosse aberto, dez estavam repetidas - disponíveis tanto online quanto em forma impressa e uma apresentava apenas o resumo, reduzindo para 61 o total de revistas analisadas.

Nessas 61 revistas foram encontrados 340 artigos que foram catalogados e analisados em dois momentos distintos, mas interligados. 0 primeiro previu o diagnóstico da quantidade de revistas nacionais na área da Educação Física e o número de artigos publicados; a região do país em que houve maior quantidade de revistas; o estrato do periódico, além do volume de artigos em cada classificação, bem como 0 ano de publicação. $O$ segundo contemplou a análise pormenorizada dos 340 artigos, com detalhamento dos tipos de ginástica abordados nos documentos; campos de atuação; vínculo dos primeiros-autores, dentre outros, em consonância com as publicações de Simões et al. (2016) e Carbinatto et al. (2016).

Vale considerar que, por se tratar de uma pesquisa realizada online, de acesso livre e público, não houve a submissão ao Comitê de Ética e nem a assinatura do Termo de Consentimento Livre e Esclarecido.

Os resultados neste artigo não contemplam todas as variáveis estudadas, visto que as publicações científicas exigem limites de palavras/página para uma determinada pesquisa e, portanto, focam apenas no diagnóstico das revistas em que os artigos foram publicados, no que tange às regiões geográficas e aos estratos em relação aos triênios de análise Web-Qualis da Capes.

\section{RESULTADOS}

Das revistas nacionais que estão catalogadas na área da Educação Física pelo sistema Web-Qualis, observamos que $11,7 \%$ do total possuem artigos sobre a temática ginástica (Quadro 1).

Quadro 1 - Total de artigos de ginástica nas revistas nacionais na área da Educação Física

\begin{tabular}{|l|c|}
\hline \multicolumn{1}{|c|}{ Revista } & № de Artigos \\
\hline Conexões & 33 \\
\hline Revista Brasileira de Ciências do Esporte & 24 \\
\hline Revista Brasileira de Nutrição Esportiva & 22 \\
\hline Pensar a Prática & 21 \\
\hline Motriz & 20 \\
\hline Revista Brasileira de Ciência e Movimento & 17 \\
\hline Movimento, Revista Mackenzie de Educação Física e Esporte, Revista de Educação Física & 15 (cada) \\
\hline Revista Brasileira de Educação Física e Esporte & 14 \\
\hline Fitness \& Performance Journal & 13 \\
\hline Revista Brasileira de Medicina do Esporte & 12 \\
\hline Revista Brasileira de Prescrição e Fisiologia do Exercício & 10 \\
\hline Revista Brasileira de Cineantropometria \& Desempenho Humano & 07 \\
\hline Movimento \& Percepção, Motrivivência, Revista Científica JOPEF & 6 (cada) \\
\hline Revista Brasileira Ciências da Saúde, Revista Brasileira de Atividade Física e Saúde, Cinergis & 5 (cada) \\
\hline Revista CPAQV & 4 \\
\hline
\end{tabular}


Continuação do Quadro 1...

Corpoconsciência, Educação Física em Revista, Revista Brasileira de Ciências do Envelhecimento Humano, Revista Inspirar, ConScientiae Saúde 3 (cada)

Arquivos de Ciências da Saúde da UNIPAR, Estudos Interdisciplinares sobre 0 Envelhecimento, Hórus (FAESO), HU Revista, Pró-Posições, Recorde: Revista de História do Esporte, Revista Brasileira de Geriatria e Gerontologia, Revista Brasileira de Saúde Ocupacional, Revista Ciência em Extensão, Revista de Nutrição, Revista Produção On line, Saúde e Pesquisa, Licere, Revista Brasileira em Promoção da Saúde, Revista Salusvita Acta Ortopédica Brasileira, Anuário da Produção Acadêmica Docente, Arquivos Sanny de Pesquisa em Saúde, Fisioterapia Brasil, O Mundo da Saúde, Revista Polêmica, Pulsar, Revista Baiana de Saúde Publica, Revista Brasileira de Ciências da Saúde, Revista Brasileira de Fisioterapia, Revista Brasileira de História da Educação, Revista Brasileira de Ortopedia, Revista Brasileira de Otorrinolaringologia, Revista Brasileira de Psicologia do

Esporte, Revista CEFAC, Revista da Sociedade Brasileira de Atividade Motora Adaptada, Revista da Sociedade Brasileira de Fonoaudiologia, Revista de Saúde Pública, Revista Gestão Industrial, Revista Latino-Americana de Enfermagem

Total - 61

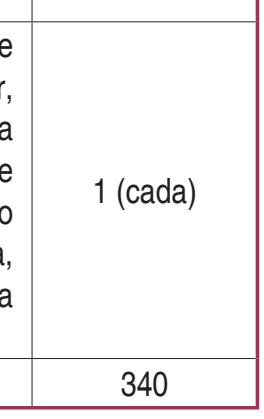

Fonte: Coleta de Dados, 2015.

Em relação à procedência territorial das revistas, identificamos que $59,1 \%$ estão lotadas na Região Sudeste; 29,5\% na Região Sul; 4,9\% na Região Centro-Oeste e 6,5\% na Região Nordeste, enquanto que na Norte não há registros. Essa realidade provavelmente se justifica em função do número de programas de pós-graduação existentes nessas regiões do país. Afinal, a referência que as regiões Sudeste e Sul ocupam no campo acadêmico é decorrente dos investimentos realizados em programas de pós-graduação, universidades e grupos de pesquisa (CARNEIRO, 2011).

Quanto aos estados da federação temos em São Paulo (30), Paraná (7), Santa Catarina (6), Rio Grande do Sul (5), Rio de Janeiro (4), Maranhão (2), Distrito Federal (2), Minas Gerais (2), Goiás (1), Ceará (1) e Bahia (1) as procedências de editoração das revistas científicas que publicaram sobre ginástica.

Observamos também um contínuo das publicações sobre o assunto, com destaque para o ano de 2010 (Gráfico 1).

Gráfico 1 - Publicações ano a ano sobre ginástica

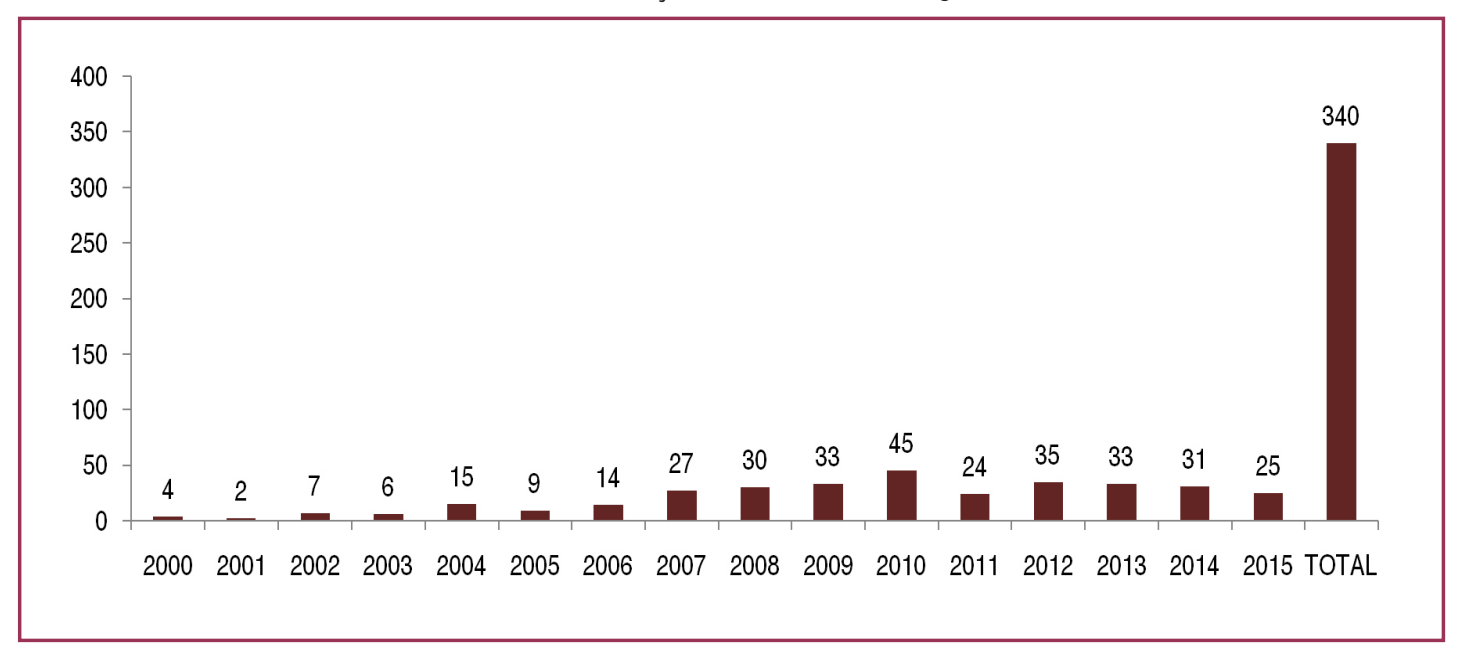

Fonte: Coleta de Dados, 2015 
Ao analisar o estrato das revistas nacionais da área da Educação Física e as relações com os artigos de ginástica encontramos que os periódicos classificados como A1 não apresentam publicações sobre o tema e os demais são estratificados de acordo com o seu ano de publicação. Assim, dos 340 artigos, foi possível analisar a estratificação de 283, pois estes foram publicados a partir de 2007, quando constatamos o início da qualificação Web-Qualis (Gráfico 2).

Gráfico 2 - Quantitativo de artigos publicados segundo o estrato da revista por período analisado

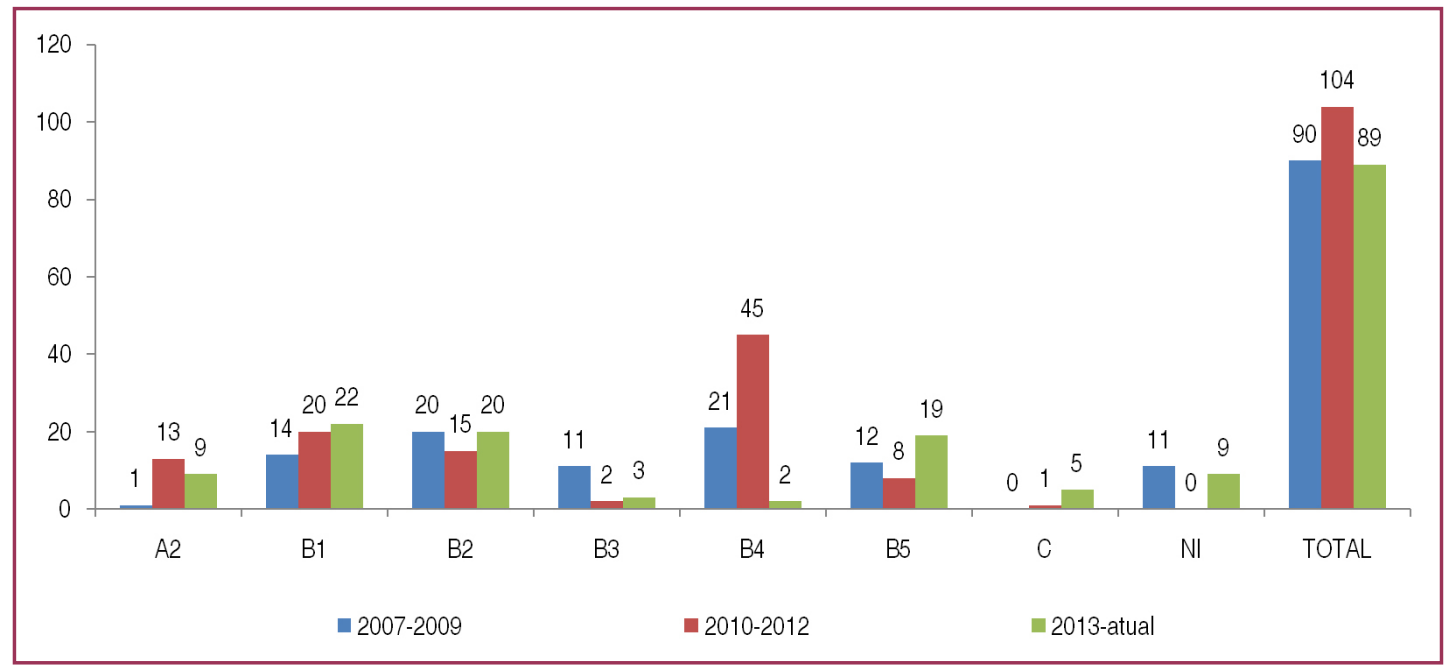

Fonte. Fonte: Coleta de Dados, 2015

Ademais, os resultados da investigação mostram que a produção do conhecimento em ginástica se dá, sobretudo, em revistas dos estratos B4, B1 e B2 respectivamente (Gráfico 3).

Gráfico 3 - Quantitativo geral dos artigos segundo o estrato da revista

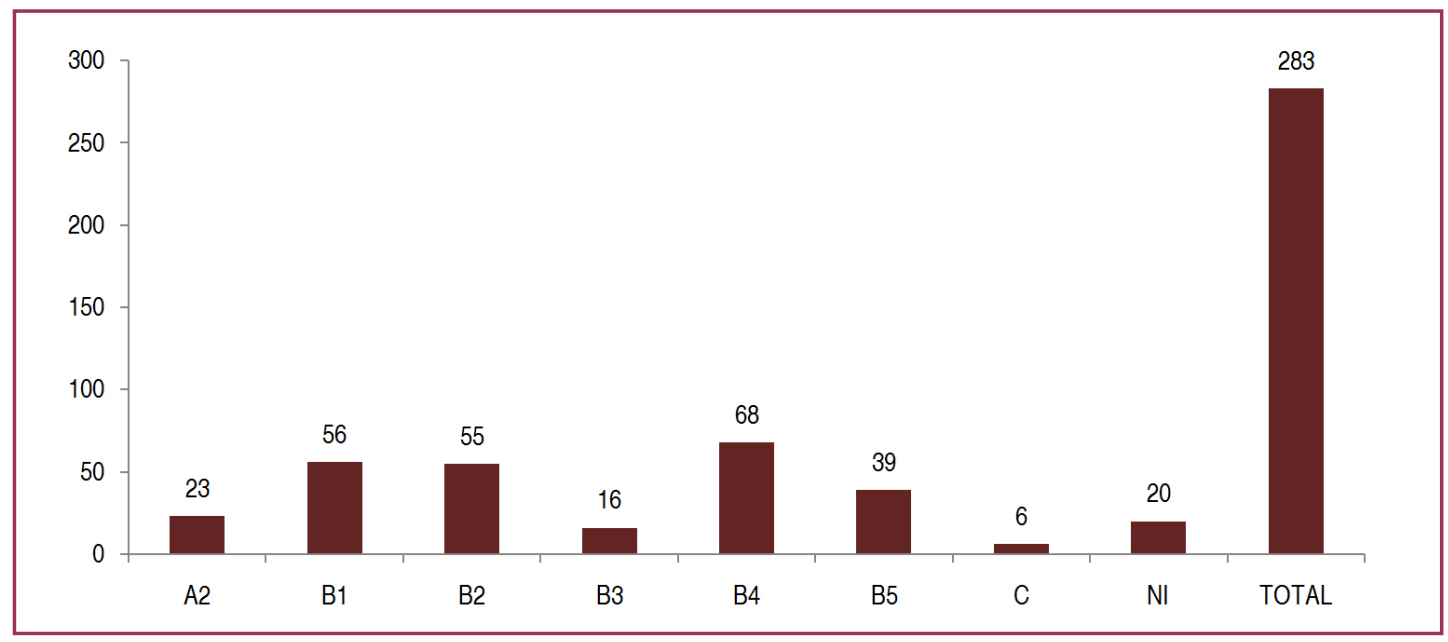

Fonte: Coleta de Dados, 2015

\section{DISCUSSÃO}

A produção de artigos científicos sobre esporte, apesar de muito menor se comparada à produção mundial, está em constante ascensão (ANDRADE et al., 2013; HELENE; RIBEIRO, 2011). Nas duas últimas décadas, o crescimento tanto quantitativo como qualitativo das 
produções em Educação Física e Esporte (KOKUBUN, 2003; TANI, 2011) foi coerente com outros movimentos na área, como o aumento do número de eventos científicos, organizações de associações específicas, periódicos especializados e publicações em periódicos de alto impacto.

Nossos resultados coincidem com essa perspectiva, ou seja, apesar de considerarmos pouco, é nítida a expansão das produções no decorrer desses 15 anos, fato corroborado por Matioli, Teixeira e Rinaldi (2014) quando analisaram 431 artigos publicados em seis periódicos no escopo A1 a B2 em Educação Física do Brasil, e notaram que 82 (19\%) pesquisas voltaramse à ginástica, e que o crescimento de publicações sobre a temática foi contínuo.

Rosa e Leta (2011) analisaram os relatórios Capes do Triênio 2001-2003 de oito dos 11 programas específicos da Educação Física ativos e avaliados pela Capes em 2004 e verificaram que as revistas que mais apresentaram publicações nos relatórios de pós-graduações (PGs) foram, respectivamente: Revista Brasileira de Ciência e Movimento (167), Motriz (124), Revista Brasileira de Medicina do Esporte (92). Assim como a investigação de Matos et al. (2013), que na análise sobre Educação Física Escolar em artigos presentes em revistas nacionais encontrou a Revista Brasileira de Ciências do Esporte, a Movimento e a Motriz como aquelas que mais publicam 0 assunto.

Provavelmente, esses são periódicos que objetivam a difusão da produção acadêmica dos pesquisadores da área de conhecimento circunscrita ao campo de intervenção da Educação Física/Ciências do Esporte, visando contribuir com a discussão e desenvolvimento da área.

Inclusive, para Folle e Nascimento (2010), a Revista Brasileira de Ciências do Esporte, revista Motus Corporis, Revista Paulista de Educação Física (atual Revista Brasileira de Educação Física e Esporte), Revista Brasileira de Ciência e Movimento, revista Movimento, revista Motrivivência, Revista Mineira de Educação Física, Revista de Educação Física/UEM, revista Motriz, revista Licere e revista Kinesis são as principais revistas da área da Educação Física em nosso país. A ginástica tem se mostrado presente em todas elas, exceto nas duas últimas (Tabela 1).

Além disso, apesar da função da universidade ser contínuo objeto de discussão no meio acadêmico (TANI, 2007), não há dúvidas da relação do tripé de ação, ensino-pesquisa-extensão. Reconhece-se que o ensino sem a pesquisa pode tornar-se provinciano, a extensão sem a pesquisa assistencialista, o ensino sem a extensão inacabado e a pesquisa sem a vinculação com o ensino e extensão descontextualizada (TANI, 2011). Não por acaso, a revista que apresenta maior produção na área - Conexões - está alocada em uma instituição (Universidade Estadual de Campinas/Unicamp) cujos trabalhos de ensino, pesquisa e extensão na ginástica são reconhecidos nacional e internacionalmente (PAOLIELLO et al., 2014). Esse fato pode, por exemplo, ampliar o número de envio de artigos sobre a temática para avaliação nessa revista.

Destacamos também o fato de a revista Conexões, usualmente, publicar números temáticos sobre "ginástica", o que alavanca a expansão na área e o perfil da revista sobre 0 tema. Ferreira Neto et al. (2003) destacam que, ao agrupar temáticas, os editores oferecem uma gama de possibilidades de pesquisa de um mesmo conhecimento, mas essa atividade deve ser dinâmica para não bloquear a revista a apenas alguns tipos de produção.

A publicação em periódicos alocados em universidades consideradas como "de pesquisa" também foi notável. Não obstante, são normalmente alocados naquelas em que há 
maior número de especialidades científicas e profissionais e onde a difusão de cursos de pósgraduação é mais reconhecida (COORDENAÇÃO DE APERFEIÇOAMENTO DE PESSOAL DE NÍVEL SUPERIOR, 2015). Coincidentemente (ou não), essas universidades são responsáveis pelo crescimento do número de eventos específicos da área, como o Fórum Internacional de Ginástica Geral (desde 2001, organizado pela Unicamp), Seminário Internacional de Ginástica Artística e Rítmica (desde 2007 e preconizado pela Unicamp, Universidade de São Paulo/USP, Universidade Estadual Paulista/Unesp) e Simpósio de Ginástica: Formação e Intervenção (2008, Universidade Estadual de Maringá/UEM).

As revistas com um ou dois artigos representaram $57,3 \%$ do total, o que denota a concentração em determinados periódicos. Tani (2014 p. 716) esclarece que a criação de periódicos "[...] não segue uma lógica definida, de modo que ela pode estar associada a uma variedade de interesses, necessidades e motivações de diferentes instituições como universidades, associações científicas, agências de fomento, editoras comerciais, entre outras". Como consequência, observamos um grande número de revistas que contemplam, em maior e menor proporção, todas as áreas de conhecimento.

Estudos sobre a ciência da informação detectam esse fenômeno como Lei da Dispersão ou Lei de Bradford (PINHEIRO, 1983; ARAÚJO, 2006; MACHADO JUNIOR et al., 2016). Criada em 1934, essa lei afere o grau de atração de periódicos a determinadas temáticas e detecta a extensão da produção de artigos científicos de uma especificidade. No caso da ginástica, em comparação ao quantitativo de periódicos e à relação de artigos publicados, nota-se um núcleo de revistas com maior prevalência de publicação. Inclusive, "[...] a aceitação e publicação destes artigos incentivam outros autores deste assunto a encaminhar seus artigos para estes periódicos" (MACHADO JUNIOR et al., 2016, p. 114).

Consideramos importante que a ampliação das produções em ginástica em revistas com maior indexação deva ocorrer concomitante com a expansão de ofertas dessas revistas com acesso livre e, inclusive, no idioma português. Afinal, como bem relatam Viveiros et al. (2015), o acesso restrito a algumas revistas e o idioma diminuem a circulação de produção entre os técnicos esportivos, por exemplo.

No Brasil, a produção de novos conhecimentos está atrelada aos programas de mestrado e doutorado e aos grupos de pesquisa vinculados a eles. Na década de 1980 há registros dos primeiros programas na área da Educação Física, liderados pela Universidade de São Paulo e Federal de Santa Maria (PEREIRA; ANDRADE; CESÁRIO, 2012), o que demonstra que cronologicamente a área ainda é incipiente. Atualmente este número é de 32 , sendo 50 cursos entre mestrados (31) e doutorados (19) acadêmicos e um profissional, dos quais 78,1\% dos programas estão na Região Sul e na Sudeste (CAPES, 2015).

Por consequência, nossos dados revelam a hegemonia das regiões Sudeste e Sul na compilação de periódicos e corroboram outros tipos de pesquisa sobre a relação da produção acadêmica e a procedência territorial (MATOS et al., 2013; MALDONADO; SILVA; MIRANDA, 2014) em relação aos artigos sobre o cotidiano escolar.

Ludorf (2002), ao analisar os resumos de teses e de dissertações em Educação Física na década de 1990, encontrou $76 \%$ na Região Sudeste, $24 \%$ na Sul e nenhuma em outras regiões. Tavares et al. (2009), buscando a produção sobre lazer, ratificaram que a Região Sul é hegemônica, seguida pelas regiões Nordeste e Sudeste, mas que tal fato se dá, sobretudo, pelas produções em outras áreas, como o turismo e o meio ambiente. 
Carbinatto e Moreira (2006) delinearam o perfil das instituições e regiões geográficas brasileiras dos artigos publicados na Revista Brasileira de Ciências do Esporte entre os anos de 2000 e 2005 e detectaram que, dos 175 artigos, 106 pertenciam a instituições públicas, sendo 83 advindos da Região Sudeste e 48 da Região Sul. Dos 43 artigos vinculados às instituições particulares, 36 pertenciam à Região Sudeste e seis à Região Sul.

Tannús e Simões (2015a) pesquisaram a temática "ginástica" em teses de doutorado nos últimos 15 anos no site da Capes, no qual foram encontradas 18 teses (13 na área da Educação Física, duas na Educação, uma na Engenharia, uma na Psicologia e uma na Alimentos e Nutrição). A maior concentração de trabalhos estava na Região Sudeste (11), seguida pela Região Sul (6) e pela Centro-Oeste (1). Em relação às dissertações de mestrado (TANNúS; SIMÕES, 2015b), foram identificadas 75 dissertações, das quais 60 na Educação Física e 15 na Educação, sendo 33 advindas de programas da Região Sudeste e 21 da Região Sul.

Se considerarmos que as pesquisas são atividades-chave para aqueles que adentram na carreira acadêmica e ampliam seu grau de formação até o seu máximo (doutorado), os nossos resultados são coerentes com a presença de um maior número de doutores na Região Sudeste $(36,7 \%)$ e na Região Sul $(33,8 \%)$. Inclusive, é por doutores que $89,1 \%$ dos grupos de pesquisas cadastrados na Plataforma Lattes são liderados (CONSELHO NACIONAL DE DESENVOLVIMENTO CIENTÍFICO E TECNOLÓGICO, 2015).

Por sua vez, os periódicos são coordenados por pesquisadores das universidades e, normalmente, atrelados à pós-graduação. Em certa medida, nossos resultados confirmam a relação direta entre procedência de periódicos em uma região e o número de publicações.

Em uma busca dos títulos de núcleos de estudos no Diretório dos Grupos de Pesquisa no Brasil (CNPq), inserimos a palavra "ginástica" e, após consulta parametrizada, identificamos 11 grupos, dos quais cinco na Região Sudeste, três na Região Sul, dois na Região Nordeste, um na Região Centro-Oeste e nenhum na Região Norte, dado que também pode justificar as editorações diferenciadas pelas regiões brasileiras.

É notável que a procedência territorial dos trabalhos coincida com a localização das revistas, que são patrocinadas por instituições localizadas em suas próprias regiões. Compreendemos que estar ou não alocado na região não é condição para envio de artigo em um determinado periódico. $O$ que queremos pontuar é que os trabalhos de determinadas universidades e grupos de pesquisadores podem influenciar na escolha e envio de material a um ou outro periódico.

Parece que o incentivo para que os periódicos nacionais adentrem em critérios mais rígidos (ANDRADE et al., 2013) tem surtido efeito, pois há melhoria das suas respectivas qualificações, o que justifica o incremento dos artigos em ginástica (Tabela 2) nos estratos A2 e B1. Ainda assim, os dados indicam que, apesar de existir periódicos em estratos considerados altos pelos padrões da produção científica brasileira, os artigos não acompanham esta realidade, pois as revistas B4 foram predominantes na produção em ginástica.

Os estudos de Maldonado, Silva e Miranda (2014) revelaram concentração de artigos sobre o cotidiano escolar na Educação Física em revistas, sobretudo, de classificação B4 $(53,6 \%), A 2(18 \%)$ e B1 (17\%), o que, de certa forma, concorda com os resultados de nossa investigação. Parece coerente dizer que há um vácuo de revistas na área nos qualis B2 e B3 para as produções nacionais, independente da temática. 
Publicar em periódicos com alta qualificação é hoje o principal objetivo dos programas de pós-graduação (SILVA; GONÇALVES-SILVA; MOREIRA, 2014). Apesar disso, Lovisolo (2007) alerta que essa situação é preocupante, pois, além da pressão que os docentes recebem dos órgãos de fomento, os processos de indexação são realizados por empresas especializadas que perspectivam o índice de impacto do material publicado e, na maioria das vezes, recebem por esse serviço. O problema se agrava quando os pesquisadores publicam em periódicos que não têm relação com a especificidade da área da Educação Física, motivados exclusivamente pelo status que isso pode gerar, o que Tani (2014, p. 718) salienta como "[...] um comportamento autocentrado na busca da produtividade a qualquer custo".

Ademais, não podemos ignorar as limitações da avaliação bibliométrica. Vitor-Costa, Maia da Silva e Soriano (2012) esclarecem que a análise de fator de impacto das revistas e o índice h dos autores (sobretudo relacionado às citações) utilizados no Brasil para avaliação da produção de pesquisa podem influenciar no distanciamento entre o que é produzido na área e a sua relevância social e acadêmica e demonstram ser de suma importância ampliar as discussões sobre a utilização da avaliação nos programas de pós-graduação em Educação Física e Esporte. Ao verificarmos as avaliações das revistas realizadas pelos programas stricto sensu dos últimos triênios percebemos que houve um aumento significativo principalmente dos estratos B2 e B4. Esse resultado pode estar associado a "[...] identidade discursiva do impresso e a sua diferenciação no conjunto de periódicos científicos da área" (SCHNEIDER et al., 2009, p. 67).

Uma preocupação presente em nossas reflexões é sobre o acesso e a vinculação da produção pela comunidade acadêmica, composta não só de pesquisadores, mas também de graduandos e de professores, por exemplo, que estão nas escolas, e que na maioria das vezes têm dificuldades de ter contato com esse material, seja por não compreender a linguagem científica, seja por não conseguir relacionar a produção de conhecimento com a sua prática pedagógica ou mesmo pela falta de tempo em função da carga horária elevada de trabalho. Aliás, após entrevistarem 205 técnicos canadenses sobre como o conhecimento produzido era transferido no seu cotidiano, Reade, Rodges e Hall (2008) perceberam que o acesso daqueles às pesquisas ainda é incipiente e desafiado pela dificuldade de conseguir tempo disponível para estudo. Talvez, trazer à tona onde se encontra a maior parte desses artigos possa sanar, ao menos num primeiro momento, essa barreira.

Apesar de a qualidade dos periódicos ser questionável (TANI, 2014), para pesquisadores que produzem na área "[...] quanto maior a credibilidade do periódico, maior a visibilidade e legitimidade concedida" (TANI, 2014, p. 716), ocorrendo a ascensão "[...] para efeito de promoção, reconhecimento e obtenção de status, posição e poder na comunidade científica".

Ao analisarmos os dados do Gráfico 1 verificamos que a distribuição anual das publicações nesses periódicos se configura no ano de 2010, representando 45 artigos. Entre 2000 e 2003 poucos estudos foram encontrados com as palavras-chave analisadas. Pontuamos duas hipóteses em relação a esse fenômeno: os meios de divulgação da produção intelectual das PGs em Educação Física nos anos de 2001 a 2003 terem sido, primordialmente em Livros e Anais de eventos, não incluídos na nossa metodologia (ROSA; LETA, 2011); e a distribuição da função docente por grau de formação de 2003 a 2013 ter saltado de 39,5\% para 53,2\% na rede pública de ensino com a ampliação considerável no regime de trabalho em tempo integral 
de docentes após o ano de 2003 (INSTITUTO NACIONAL DE ESTUDOS E PESQUISAS EDUCACIONAIS ANÍSIO TEIXEIRA, 2013).

Em relação aos estratos (Gráfico 2 e Gráfico 3), o destaque é para o Qualis B4 no ano de 2012, o qual mostrou o maior número de artigos (19), sendo a revista Conexões aquela com alta produção no ano (15).

Por outro lado, os periódicos melhor classificados (A2) só apresentaram publicações a partir do ano de 2009. Gostaríamos de salientar que nenhuma das revistas publicou continuamente estudos de ginástica no período analisado.

Ousamos elencar que a continuidade na elevação de publicações na ginástica coincide com os eventos na área. A investigação de Artusi (2008) apontou esse crescimento no Brasil, especialmente a partir do final do século passado. Na Ginástica Para Todos encontramos I e II Encontros de Ginástica Geral (1996 e 1999); Fórum Brasileiro de Ginástica Geral (1999); I Fórum Estadual de Ginástica Geral em 2002; I ao VII Fóruns Internacionais de Ginástica Geral (2001, 2003, 2005, 2007, 2009, 2011 e 2014); Seminário Internacional de Ginástica Artística e Rítmica de Competição (2007, 2010, 2012 e 2015) e o Simpósio de Ginástica- Formação e Intervenção em 2008. Salvo este último evento, que aconteceu em Maringá/Paraná, todos os demais eventos foram no estado de São Paulo.

A diminuição do quantitativo de publicação após o ano de 2010 pode ser explicada pela busca da internacionalização das pesquisas, sobretudo após o ano de 2008. Andrade et al. (2013) indicam que após 0 ano de 2010 houve um aumento considerável das publicações em revistas internacionais. No Brasil, o salto foi de 260 publicações no quinquênio 2003-2007 para 1665 em 2008-2012.

A busca pormenorizada realizada na revista "Science of Gymnastics" - a única especializada na área e reconhecida pela Federação Internacional de Ginástica (FIG) demonstra que, a partir de 2011, ao menos um artigo publicado é de pesquisador e procedência de pesquisa brasileira.

Claro está que não excluímos a limitação de nossos estudos em relação a não abrangência de periódicos internacionais, considerando o avanço das produções neste quesito nos últimos tempos (KOKUBUN, 2006) e o fato de que a produção científica no Brasil nas Ciências Humanas apresenta um número de livros e capítulos significativamente maior do que de artigos. Esse dado ratifica que as publicações de ginástica podem ser ampliadas além do que foi encontrado nesta investigação (PEREIRA; ANDRADE; CESÁRIO, 2012).

Ainda assim, apesar de o Brasil se destacar nas produções internacionais dos países da América do Sul no levantamento entre 1970 e 2012 na base de dados Web of Knowledge, a maioria (63\%) delas é publicada em periódicos nacionais (ANDRADE et al., 2013), o que confirma que nossa pesquisa pode trazer um importante retrato da realidade sobre a ginástica.

\section{CONSIDERAÇÕES FINAIS}

Analisar os periódicos da área, de acordo com Sampaio (2014, p. 734), é "[...] simultaneamente responsabilidade e oportunidade de compartilhar uma leitura de realidade, um ponto de vista, um lugar de onde se organiza a reflexão, um conjunto de perguntas sobre a realidade, certamente outros olhares e dizeres." 
Ampliar o número de artigos sobre ginástica não garante a qualidade e reconhecimento dos trabalhos nacionais pela comunidade científica. Exemplo disso é que entre os anos de 1970 e 2012, nas bases de dados pertencentes ao Thomson Reuters, o Brasil é - disparadamente - o país com maior quantitativo de artigos, mas o fator de impacto é bem menor do que o do Peru, Uruguai , Bolívia e Chile. Portanto, não deixa de ser limitador o fato de apresentarmos dados bibliográficos quantitativos que não excluem a possibilidade de nos dar indícios sobre 0 tema (ANDRADE et al., 2013).

Não defendemos apenas o aumento numérico das produções, mas também a expansão da qualidade e reconhecimento dos nossos periódicos na comunidade da Educação Física e Esporte. Devemos atentar: se os pesquisadores do nosso país buscarem apenas revistas ranqueadas e de impacto internacional já reconhecido, corremos o risco de não fortalecermos os periódicos nacionais (SAMPAIO, 2014) e por isso todo cuidado é necessário nas críticas sobre 0 assunto. Tani (2014) sublinha que 0 crescimento de pesquisadores que publicam artigos em extratos A1 em áreas correlatas à Educação Física provoca a elevação da mediana do fator de impacto daqueles e, como resultado, distancia os periódicos específicos da área que têm dificuldade em elevar seu prestígio.

$O$ incentivo para a internacionalização induz à formatação do trabalho em outros idiomas, sobretudo o inglês (VAZ; ALMEIRA; BASSANI, 2015), e em revistas com acesso restrito, o que pode dificultar a aquisição dos resultados por parte de profissionais da área.

\section{REFERÊNCIAS}

ANDRADE, David et al. Bibliometric analysis of South American research in sports science from 1970 to 2012. Motriz, v. 19, n. 4, p. 783-791, 2013.

ARAUJO, Carlos Alberto. Bibliometria: evolução histórica e questões atuais. Em Questão, v. 12, n. 1, p. 11-32, 2006.

ARTUSI, Maryland. Diagnóstico dos principais eventos de Ginástica Geral. 2008. 228 f. Dissertação (Mestrado) - Curso de Educação Física, Universidade São Judas Tadeu, São Paulo, 2008. Disponível em: <http://www.usjt.br/biblioteca/mono disser/mono diss/082.pdf >. Acesso em: 15 dez. 2015.

BASKURT, Oguz. Time series analysis of publication counts of a university: what are the implications? Scientometrics, v. 86, n. 3, p. 645-656, 2011.

BETTI, Mauro et al. A avaliação da Educação Física em debate: implicações para a subárea pedagógica e sociocultural. Revista Brasileira de Pós-Graduação, v. 1, p. 183-194, 2004.

COORDENAÇÃO DE APERFEIÇOAMENTO DE PESSOAL DE NÍVEL SUPERIOR. Web Qualis da CAPES. 2012. Disponível em: <http://www.periodicos.capes.gov.br>. Acesso em: 30 abr. 2015.

COORDENAÇÃO DE APERFEIÇOAMENTO DE PESSOAL DE NÍVEL SUPERIOR. Avaliação Trienal 2013. 2015. Disponível em: <http://www.capes.gov.br>. Acesso em: 2 ago. 2015.

CARBINATTO, Michele; MOREIRA, Wagner Wey. Produção científica em Educação Física e regiões geográficas brasileiras: 0 caso da revista do CBCE. Revista Brasileira de Educação Física e Esporte, v. 20, p. 342-342, 2006. 
CARBINATTO, Michele; MOREIRA, Wagner Wey; CHAVES, Aline Dessupoio; SANTOS, Suziane Peixoto; SIMÕES, Regina Rovigati. Campos de Atuação em Ginástica: estado da arte nos periódicos brasileiros. Movimento, v.22, n.3, p. 917-928, 2016.

CARNEIRO, Felipe Ferreira Barros. Políticas científicas em educação física: a arqueologia do GTT Escola no Congresso Brasileiro de Ciências do Esporte (1997-2009). 2011. 162 f. Dissertação (Mestrado) - Programa de Pós-Graduação em Educação Física, Universidade Federal do Espírito Santo, Vitória, 2011.

CONSELHO NACIONAL DE DESENVOLVIMENTO CIENTÍFICO E TECNOLÓGICO. Censos de Grupos de Pesquisas. 2015. Disponível em: <http://lattes.cnpq.br/web/dgp/sobre14>. Acesso em: 10 ago. 2015.

ERCAN, Gonenc; CICEKLI, llyas. Using lexical chains for keyword extraction. Information Processing and Management, v. 43, p. 1705-1714, 2007.

FERREIRA, Fernanda; TEIXEIRA, David. Atualidade da produção científica brasileira sobre o ensino da ginástica para pessoas com deficiência: contribuições para Educação Física escolar. In: FÓRUM INTERNACIONAL DE GINÁSTICA GERAL, 7., Campinas, 2014. Anais... São Paulo, 2014. p. 283287.

FERREIRA NETO, Amarílio. Catálogo de periódicos de Educação Física e esportes (1930-2000). Vitória: Proteoria, 2002.

FERREIRA NETO, Amarílio et al. A Revista Brasileira de Ciências do Esporte e os 25 anos de CBCE. Revista Brasileira de Ciências do Esporte, v. 1, n. 1, p. 1-25, 2003.

FOLLE, Alexandra; NASCIMENTO, Juarez Vieira. Trajetória docente em educação física: percursos formativos e profissionais. Revista Brasileira de Educação Física e Esportes, v. 24, n. 4, p. 507523, out./dez. 2010.

GOKCEOGLU, Candan; OKAY Aral; SEZER, Ebru. International earth science literature from Turkey 1970-2005: trends and possible causes. Scientometrics, v. 74, n. 3, p. 409-423, 2008.

HELENE, André; RIBEIRO, Pedro. Brazilian scientific production, financial support, established investigators and doctoral graduates. Scientometrics, v. 89, n. 2, 677-686, 2011.

INSTITUTO NACIONAL DE ESTUDOS E PESQUISAS EDUCACIONAIS ANÍSIO TEIXEIRA. Censo da educação superior (CENSUP). 2013. Disponível em: < http://download.inep.gov.br/download/ superior/censo/2013/resumo tecnico censo educacao superior 2013.pdf>. Acesso em: $20 \mathrm{dez}$. 2015.

KOKUBUN, Eduardo. Pós-graduação em Educação Física no Brasil: indicadores objetivos dos desafios e das perspectivas. Revista Brasileira de Ciências do Esporte, v. 24, n. 2, p. 9-26, 2003.

KOKUBUN, Eduardo. Pós-graduação em Educação Física. Revista Brasileira de Educação Física e Esporte, v. 20, n. 5, p. 31-33, 2006.

LIMA, Letícia et al. UNESP-UNICAMP-USP: A produção científica sobre a ginástica artística e rítmica de competição na pós-graduação. Revista Brasileira de Educação Física e Esporte, v. 29, n. 8, p. R60, 2015.

LOVISOLO, Hugo. Levantando o sarrafo ou dando tiro no pé: critérios de avaliação e qualis das pósgraduações em Educação Física. Revista Brasileira de Ciências do Esporte, v. 29, n. 1, p. 23-33, 2007.

LÜDORF, Sílvia. Panorama da pesquisa em educação física da década de 90: análise dos resumos de dissertações e teses. Revista da Educação Física/UEM, v. 13, n. 2, p. 19-25, 2002. 
MACHADO JUNIOR, Celso et al. As leis da bibliometria em diferentes bases de dados científicos. Revista de Ciências da Administração, v. 18, n. 44, p. 111-123, 2016.

MALDONADO, Daniel; SILVA, Sheila; MIRANDA, Maria. Pesquisas sobre a Educação Física no cotidiano da escola: 0 estado da arte. Movimento, v. 20, n. 4, p. 1373-1395, 2014.

MATIOLI, Jonas S.; TEIXEIRA, Fabiane C.; RINALDI, leda Parra B. A ginástica no Brasil: apontamentos sobre a Produção do conhecimento. In: BORTOLETO, Marco Antonio Coelho et al. FÓRUM INTERNACIONAL DE GINÁSTICA GERAL, 7. 2014. Campinas, SP: UNICAMP/FEF: SESC, 2014. p. 93-99.

MATOS, Juliana et al. A produção acadêmica sobre conteúdos de ensino na Educação Física escolar. Movimento, v. 19, n. 2, p. 123-148, 2013.

METODOLOGIA do ensino de Educação Física. São Paulo: Cortez, 1992.

MILANI, Camila; SOARES, Daniele; BORTOLETO, Marco. Ginástica: a produção dos estudantes de graduação e especialização da faculdade de Educação Física da UNICAMP 1985-2014. Coleção Pesquisa em Educação Física, v. 14, n.3, p. 89-98, 2015.

MUGNAINI, Rogério; CARVALHO, Telma; CAMPANATTI-ORTIZ, Heliane. Indicadores de produção científica: uma discussão conceitual. In: POBLACIÓN, Dinah; WITTER, Geraldina; SILVA, José (Org.). Comunicação e produção científica: contexto, indicadores, avaliação. São Paulo: Angellara, 2006. p. 313-340.

PAOLIELLO, Elizabeth et al. Grupo Ginástico Unicamp: 25 anos. Campinas: Editora Unicamp, 2014.

PEREIRA, Ana; ANDRADE Thaís; CESÁRIO Marilene. A produção do conhecimento científico em ginástica. Conexões, v. 10, p. 56-79, 2012. N. esp.

PINHEIRO, Lena Vânia Ribeiro. Lei de Bradford: uma reformulação conceitual. Ciência da Informação,v. 12, n. 2, p. 59-80, 1983.

READE lan; RODGERS Wendy; HALL, Nathan. Knowledge transfer: how do high performance coaches access the knowledge of sport scientists. International Journal of Sports Science and Coaching, v. 3, p. 319-334, 2008.

RINALDI, leda Parra Barbosa. A ginástica como área de conhecimento na formação profissional em Educação Física: encaminhamentos para uma reestruturação curricular. 2005. 232 f. Tese (Doutorado) - Programa de Pós-Graduação em Educação Física, Universidade Estadual de Campinas, Campinas, 2005.

ROSA, Suely; LETA, Jacqueline. Tendências atuais da pesquisa brasileira em Educação Física: parte 1: uma análise a partir de periódicos nacionais. Revista Brasileira de Educação Física e Esporte, v. 24, n.1, p. 121-134, 2010.

ROSA, Suely; LETA, Jacqueline. Tendências atuais da pesquisa brasileira em Educação Física. Parte 2: a heterogeneidade epistemológica nos programas de pós-graduação. Revista Brasileira de Educação Física e Esporte, v. 25, n. 1, p. 7-18, 2011.

SAMPAIO, Gabriela; KRAESKI, Ana; FARIAS, G. O. Análise da produção científica relacionada a Ginástica Rítmica na base de dados LILACS. Revista Brasileira de Educação Física e Esporte, v. 29, n. 8, p. R41, 2015.

SAMPAIO, Tania. Desafios e perspectivas para a divulgação da pesquisa em Educação Física: um processo de construção. Revista Brasileira de Ciência do Esporte, v. 36, n. 4, p. 733-739, 2014. 
SCHNEIDER, Omar et al. Arqueologia das práticas editoriais: 15 anos de um impresso em Movimento. Movimento, v. 15, n. 3, p. 57-85, jul./set. 2009.

SILVA, Deisy et al. 0 estado da arte da ginástica nos Anais do Fórum Internacional de Ginástica Geral de 2001 a 2012. Conexões, v. 13, p. 211-229, 2015.

SILVA, Junior Vagner Pereira da; GONÇALVES-SILVA, Luiza Lana; MOREIRA, Wagner Wey. Produtivismo na pós-graduação. Nada é tão ruim, que não possa piorar. É chegada a vez dos orientandos! Movimento, v. 20, n. 4, p. 1423-1445, 2014.

SIMÕES, Regina et al. A produção acadêmica sobre ginástica: estado da arte dos artigos científicos. Revista Brasileira de Educação Física e Esporte, v. 30, p. 183-198, 2016.

SOUZA, Juliano; MARCHI JUNIOR, Wanderley. Por uma sociologia da produção científica no campo acadêmico da Educação Física no Brasil. Motriz, v. 17, n. 2, p. 349-360, 2011.

TANI, Go. Avaliação das condições do ensino de graduação em educação física: garantia de uma formação de qualidade. Revista Mackenzie de Educação Física e Esporte, v. 6, n. 2, p. 55-70, 2007.

TANI, Go. A Educação Física e o esporte no contexto da universidade. Revista Brasileira de Educação Física e Esporte, São Paulo, v. 25, p. 117-126, 2011.

TANI, Go. Editoração de periódicos em Educação Física/Ciências do Esporte: dificuldades e desafios. Revista Brasileira de Ciências do Esporte, v. 36, n. 4, p. 715-722, 2014.

TANNÚS, Fernanda; SIMÕES, Regina. Ginástica: a produção científica nas teses de doutorado. Revista Brasileira de Educação Física e Esporte, v. 29, n. 8, p. R57, 2015a.

TANNÚS, Fernanda; SIMÕES, Regina. O estado da arte nas dissertações de mestrado da Educação e Educação Física: o caso da ginástica. Revista Brasileira de Educação Física e Esporte, v. 29, n.8, p. R57, 2015b.

TAVARES, Giselle et al. Gestão do lazer: os grupos de pesquisa em foco. Motriz, v. 15, n. 3 p. 470 480, 2009.

TIBEAU, Cynthia C. Pasqua Mayer. Diferentes olhares sobre a Ginástica Geral. In: FÓRUM BRASILEIRO DE GINÁSTICA GERAL, 1999. Campinas. Anais... Campinas: Unicamp:SESC, 1999. v. 1 , p. $57-62$

TONELLO, Izângela; LUNARDELLI, Rosane Alves; ALMEIDA JUNIOR, Oswaldo Francisco. Palavraschave: possibilidades de mediação da informação. Ponto de Acesso, v. 6, p. 21-34, 2012.

VAZ, Alexandre; ALMEIDA, Felipe; BASSANI, Jaison. Novas demandas aos periódicos, assim como à comunidade acadêmica. Revista Brasileira de Ciências do Esporte, v. 37, n. 1, p. 1, 2015.

VITOR-COSTA, Marcelo; MAIA DA SILVA, Priscila; SORIANO, Jeane Barcelos. A avaliação da produtividade em pesquisa na Educação Física: reflexões sobre algumas limitações dos indicadores bibliométricos. Revista Brasileira de Educação Física e Esporte, v. 26, n. 4, p. 581-97, 2012.

VIVEIROS, Luís et al. Ciência do Esporte no Brasil: reflexões sobre o desenvolvimento das pesquisas, o cenário atual e as perspectivas futuras. Revista Brasileira de Educação Física e Esporte, v. 29, n. 1, p. 163-175, 2015. 


\section{Apoios:}

Conselho Nacional de Pesquisa e Desenvolvimento Científico e Tecnológico (CNPq) Processo n. 472119/2014-1 e Fundo de Amparo à Pesquisa do Estado de Minas Gerais (FAPEMIG). 